\title{
Smart Fashion and Wearable Technology: Opportunities and Challenges for Future Growth
}

\author{
Su Yun Bae* and Deborah G Wooldridge \\ Bowling Green State University, USA
}

*Corresponding author: Su Yun Bae, Apparel Merchandising and Product Development, Bowling Green State University, Bowling Green, USA.

Received Date: October 09, 2018

Published Date: October 17, 2018

\begin{abstract}
Technology advances have driven innovations within various industries and supported various aspects of humans' lives. Technology has also impacted the fashion industry in terms of its design and production processes. Primary examples of innovations include 3D printing, smart textiles, and smart wearable devices. The major issue related to technology in fashion is centered around its future growth. This paper reviews each of the innovative technologies and their usability, functionality, and affordability as opportunities and challenges toward commercialization.
\end{abstract}

Keywords: 3D printed fashion; Technology commercialization; Smart textiles; Smart wearable technology

\section{Introduction}

The continuous advances in technology have facilitated innovations within various industries and supported various aspects of humans' lives. Consumers are living in the era of a significant transition from simply utilizing the technology to incorporating it into every aspect of their daily lives. The way of communicating with other people has been smarter, and lifestyle has been impacted by new technologies such as iRobot Roomba and Google Home. The fashion industry has also become smarter with the assistance of technology. Significant innovations related to fashion goods include three-dimensional (3D) printing on fashion design, smart textiles, and smartwatch and jewelry. This paper reviews each of these innovations along with their opportunities and challenges for future profitability.

\section{Discussion}

Technological advances in manufacturing and textile production have affected the creation of fashion design. Technology itself has innovated fashion as well. Brands who have taken fashion to the next level include Chanel, Katya Leonovich, Victoria's Secret, and Apple to name a few. Although the application of technology in fashion is in its preliminary stage of innovation, it will continuously advance and change consumers' perceptions and preferences. Primary examples of fashion innovations are discussed below, followed by the opportunities and challenges of each innovation.

\section{D printed fashion}

$3 \mathrm{D}$ printing is an additive manufacturing technology that creates a three-dimensional object by setting down successive layers of material until it develops a complete form [1]. Instead of creating flexible garments, the process is more useful to create rigid and geometric shapes. Although 3D printing facilitates creativity in fashion design due to its unique shape and rigidity, the process has hindered its commercialization due to the significant printing time and high skills required for the production. The unique quality of rigid structure poses functional limitations of garments. Designers have built 3D printed garments through meshes to overcome the rigidity and improves flexibility [2]. Danit Peleg is one of the fashion designers who were able to commercialize her design. The designer is selling 3D printed bomber jacket for $\$ 1,500$ on her website. The material still limits the designer to sell other types of garments such as bottoms or dresses to the public. In addition to the challenge related to flexibility, other challenges include its durability and cost factor. There is a tremendous opportunity in 3D printed clothing, but this technology can expect more immediate success in other aspects of apparel such as jewelry, accessories, and athletic wear to improve energy absorption and stiffness for padding and shoe soles [3]. 3D printed fashion can also be marketed as a sustainable option considering its zero-waste creation and minimized production impact on the environment. 


\section{Smart textiles}

Smart textiles can be categorized into two broad types. One focuses more on aesthetic applications for smart textiles while the other puts more importance on its increased functionality [4]. Aesthetics of smart textiles are mainly created by LED lights that are embedded onto textiles. Katy Perry was one of the celebrities who wore an LED gown at a special event. Chanel also created handbags with LED lights for its 2017 Spring and Summer collection and the selling prices ranged from $\$ 7,000$ to $\$ 9,000$. The application of LEDs or electroluminescent materials is more plausible in high-end fashion due to high production cost. However, it can be applied to home goods such as curtains to light up rooms. A more complicated version for smart textiles is the technology that senses and responds to physical and environmental stimuli such as temperature, heat, and biometric data. Smart fabrics react to external information by regulating body temperature, vibrating muscles, and releasing medication or moisture into humans' skin [4]. Smart textiles propelled by human-centered technologies pose great potential in the medical and athletic fields [5].

\section{Smart watch and jewelry}

Smartwatch and jewelry have been much more commercialized than any other smart fashion due to its relatively simpler technological application and the prevalence of digital technology. As a leading company in the smart watch sector, Apple has successfully commercialized the technology through a high but effective pricing strategy and collaborations with high-end brands like Hermès that help present its creativity and innovativeness. Such technology can be simple like Apple Watch or Fitbit or more sophisticated like watch and jewelry that gather physiological data or sense emotions. For example, Empatica is selling wearable devices that record physiological signals and they help track seizures and assist other minor and significant health problems from sleep disorders, addiction, and depression to Alzheimer's and Diabetes, to name a few. Feel, the first emotion tracker is a similar technology but tracks five key emotions such as joy, contentment, sadness, tension, and distress. This technology helps process and regulate emotions and support mental health, offering great profit potential in various consumer markets. Through its collaboration with the Internet of Things and Artificial Intelligence, this technology can not only improve humans' daily lives by automatically playing music depending on moods and but also increase company profitability by marketing their products and services aligning with real-time sensed feelings and emotions.

\section{Conclusion}

Technology has continuously impacted the practice of fashion design and production. Although fashion and technology have always intersected, computational advances and technological innovations have enabled the adoption of new approaches to design. Thus, advances in design will continue in a much smarter way. Such technological advances have posed tremendous opportunities for future growth in fashion. However, designers and their collaborators need to find solutions for related challenges to improve its usability, functionality, and affordability. Though the target markets of fashion products inspired by technology are primarily early adopters or those with special needs, it is crucial to enhance the commercialization of those products. This is because the adoption by mass consumers facilitates the process of usercentric innovations and advancements.

\section{References}

1. Vanderploeg A, Lee SE, Mamp M (2017) The application of 3D printing technology in the fashion industry. Intl J Fashion Des Technol Educ 10(2): 170-179.

2. 3D printed fashion: why is additive manufacturing interesting for fashion?

3. Jacobson R (2017) The shattering truth of 3D-printed clothing.

4. Syduzzaman M, Patwary SU, Farhana K, Ahmed S (2015) Smart textiles and nano-technology: a general overview. J Textile Sci Eng 5(1): 181.

5. Fu W (2018) Modern fibers: a human- centered technology. J Textile Sci Fashion Tech 1(2): 1 . 